\title{
Inversion-symmetry breaking in spin patterns by a weak magnetic field
}

\author{
I. Krešić, ${ }^{1,2,3,{ }^{*}}$ G. R. M. Robb, ${ }^{1}$ G. Labeyrie, ${ }^{4}$ R. Kaiser, ${ }^{4}$ and T. Ackemann ${ }^{1}$ \\ ${ }^{1}$ SUPA and Department of Physics, University of Strathclyde, 107 Rottenrow East, Glasgow G4 ONG, United Kingdom \\ ${ }^{2}$ Institute of Physics, Bijenička cesta 46, 10000 Zagreb, Croatia \\ ${ }^{3}$ Institute of Theoretical Physics, Vienna University of Technology, Vienna A-1040, Austria \\ ${ }^{4}$ Université Côte d'Azur, CNRS, Institut de Physique de Nice, 06560 Valbonne, France
}

(Received 26 February 2019; published 30 May 2019)

\begin{abstract}
Laser-driven cold atoms near a plane retroreflecting mirror exhibit self-organization above a pump threshold. We analyze the properties of self-organized spin patterns in the ground state of cold rubidium atoms. Antiferromagnetic patterns in zero magnetic field give way to ferrimagnetic patterns if a small longitudinal field is applied. We demonstrate how the experimental system can be modeled as spin-1 atoms diffractively coupled by the light reflected by the mirror. The roles of both dipolar and quadrupolar magnetization components in determining the threshold and symmetry variations with a weak longitudinal magnetic field are examined. Although the magnetic structures correspond dominantly to a lattice of magnetic dipoles, the symmetry breaking to ferrimagnetic structures in a finite field is mediated by the coupling to a homogenous quadrupole (alignment), which is not possible in a spin-1/2 system. Our study provides a basis for further exploration of instabilities in driven multilevel systems with feedback.
\end{abstract}

DOI: 10.1103/PhysRevA.99.053851

\section{INTRODUCTION}

Light-mediated cold-atom self-organization is an emerging research avenue with potential applications in metrology and condensed matter simulation [1-19]. In this paper, we study the phenomenon of self-organization arising in an optically nonlinear sample due to diffractive coupling via single mirror feedback $[20,21]$. While initial observations of these structures were performed in liquid crystals and warm atomic vapors $[22,23]$, the scheme was recently extended to thermal cold gases, where the nonlinearity can be of optomechanical, electronic saturable, or magnetic origin, with corresponding structuring of atomic density, optical coherence, and magnetization, respectively [5,6,24-26]. These systems are interesting as they have a single pump axis and hence allow for spontaneous symmetry breaking of the translational and rotational degrees of freedom in the plane transverse to this axis, whereas in systems with multiple distinguished axes (e.g., a cavity axis and a pump axis), the potential symmetries and realizations are constrained.

As in Refs. [25,26], in this article the relevant degrees of freedom are populations and coherences in the ground-state Zeeman sublevels and the optical nonlinearity is provided by optical pumping, i.e., is magnetic optical in origin. The resulting instability creates both transverse spatial modulations of the atomic spins and the polarization profile of the laser beam.

The magnetic phase space of the instabilities was explored in Ref. [26]. In zero magnetic field, complementary intensity patterns with square symmetry are found in the $\sigma^{ \pm}$components of the transmitted beam. These are optical spin patterns arising from spontaneous symmetry breaking of the zero net

\footnotetext{
*ikresic@ifs.hr
}

optical spin state in the linearly polarized pump field. The optical patterns indicate the spontaneous emergence of a spin pattern in the atoms, i.e., a spontaneous magnetic ordering of dipoles, in this case antiferromagnetic. In Ref. [25], an analogy was established between this system and the Ising model. Diffractive ripples in the feedback field caused by a local perturbation of the atomic magnetization lead to optical pumping in the same direction one lattice period away and opposite direction half a lattice period away, leading to the antiferromagnetic coupling. If the up-down symmetry is broken by a small longitudinal field, the antiferromagnetic spin phase gives way to ferrimagnetic phases with hexagonal order. These became more irregular at higher absolute values of the magnetic field. In Refs. $[25,26]$, it was hypothesized that the symmetry breaking at large $\left|B_{z}\right|$ is due to the linear and the incoherent nonlinear Faraday effect, but that at small $\left|B_{z}\right|$ the coupling of the dipole states to higher multipoles via coherent effects is important. In this article, we provide a systematic study of the variation of pattern properties with the longitudinal magnetic field $B_{z}$, outlined in Ref. [25]. The threshold and symmetry properties are studied both experimentally and theoretically, with one of the main goals being the elucidation of the pattern selection and symmetry-breaking mechanism.

Previous modeling of transverse nonlinear polarization instabilities in laser-driven atomic media focused either on spin-1/2 ground states [27-31] or solely on the electric field evolution, eliminating the medium dynamics [32-34]. We model the experimental transition by $F=1 \rightarrow F^{\prime}=2$, which is a minimal model for $F \rightarrow F+1$ transitions, allowing for atomic quadrupoles, where $F \geqslant 1$ is an integer [35]. We show that the modeling of spin pumping processes by an effective spin- $1 / 2$ ground state, including only rate equations for the spin dipoles, is insufficient to describe the variation of pattern properties with an applied longitudinal magnetic field 
$B_{z}$ and how this is amended by using instead the optical Bloch equations, including both populations and coherences in the ground states of a spin-1 model. We calculate analytically the expressions for the threshold and the pattern symmetry parameter at a given $B_{z}$, by using the experimentally motivated approximation that the instability is driven by optical pumping of atomic spins due to an intensity difference in the two $\sigma$ components of the feedback field. Moreover, another result of this analysis is the discovery that the inversion, i.e., updown, symmetry of the system is broken at small $\left|B_{z}\right|$ by a coherent nonlinear Faraday effect, governed by light-induced $|\Delta m|=2$ ground-state coherences [36]. We demonstrate that the threshold dynamics of the spin perturbation amplitudes in the simplified model is determined by a set of complex Ginzburg-Landau equations [37], describing wave mixing of spin modes on a Talbot circle. The symmetry breaking is provided in these equations by a term quadratic in the spin modes.

\section{EXPERIMENTAL SETUP}

Most of the experimental data presented in this paper were obtained with a setup located at the University of Strathclyde. A cloud of $N=9 \times 10^{8}{ }^{87} \mathrm{Rb}$ atoms at $T=125 \mu \mathrm{K}$ is loaded into a magneto-optical trap (MOT), which is then released by turning off the cooling laser beams and the gradient magnetic field, after which an external homogeneous magnetic field is applied to facilitate the study of pattern properties. After a waiting time of $3.5 \mathrm{~ms}$ needed for stray magnetic fields to decay, a cloud with on-resonance optical thickness of about $b_{0}=27$ and FWHM $\approx 3 \mathrm{~mm}$ is prepared for pattern formation experiments. A linearly polarized pump beam with a FWHM of $0.8 \mathrm{~mm}$, intensities in the range $I=1-30 \mathrm{~mW} / \mathrm{cm}^{2}$, and a typical detuning of $\Delta=-7 \Gamma_{1}=-14 \Gamma_{2}$, where $\Gamma_{1}$ is the population and $\Gamma_{2}$ is the optical coherence decay rate, is then turned on to irradiate the center of the cloud for a typical duration of $\Delta t=250 \mu \mathrm{s}$. This pattern-inducing pump beam is retroreflected from a feedback mirror with reflectivity $R=$ 0.95. The mirror is put (i.e., imaged) at an effective distance $d$ of a few millimeters from the center of the cloud by using a pair of lenses with focal lengths $f=12.5 \mathrm{~cm}$ placed between the cloud and mirror in the $4 f$ configuration [38]. The small part of the light transmitted through the mirror is used for pattern imaging of the $\sigma$-polarization components. Both real space or near-field (NF) images of the reentrant beam intensity distributions and Fourier space or far-field (FF) data are used in the results presented in this paper. A simplified schematics of the setup is presented in Fig. 1 .

Additional observations were done in a setup at the Université Côte d'Azur, described in Refs. [25,26]. The main difference to the setup described above is that a higher optical density of up to 110 can be obtained.

\section{EXPERIMENTAL OBSERVATIONS}

\section{A. Pattern symmetries}

In Fig. 2, we present NF images of experimental realizations of patterns characteristic for the three ranges of the applied longitudinal magnetic field, $B_{z}$. Near $B_{z}=0$, patterns with square and rectangular symmetries were observed. In the Strathclyde setup, these patterns contained defects, deforma-

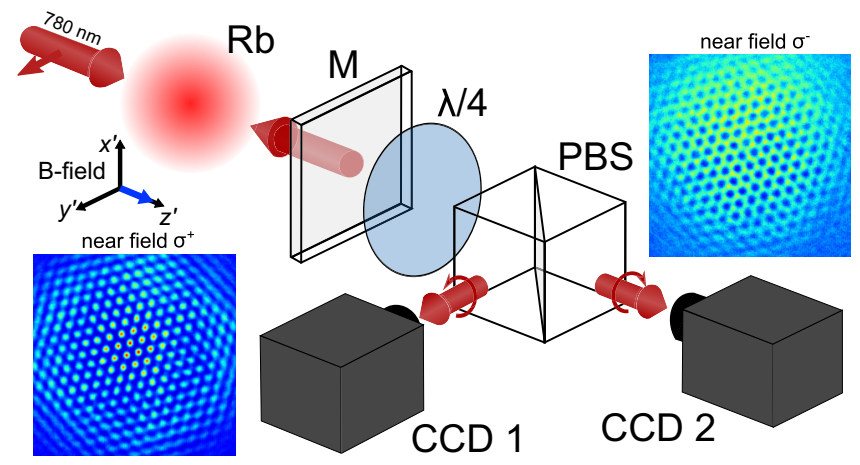

FIG. 1. Simplified schematics of the experimental setup, adapted from Ref. [25]. A linearly polarized pump beam is reflected from a feedback mirror to drive the spin self-organization in the atomic cloud. A small transmitted part of the beam is used for polarization selective NF and FF (not shown) imaging. Inversion symmetry is broken by applying a small longitudinal magnetic field (B field), resulting in formation of hexagons and honeycombs in the $\sigma$ polarization channels (inset: NF data). Rb, cold cloud of ${ }^{87} \mathrm{Rb} ; \mathrm{M}$, feedback mirror; $\lambda / 4$, quarter-wave plate; PBS, polarizing beamsplitter cube; CCD, charge-coupled device camera.

tions, and irregularities of amplitudes [see center column of Fig. 2(a)], the analysis of which, although in itself interesting, is beyond the scope of this article. For the Nice setup, a clear long-range order with square symmetry was observed.

Increasing the $\left|B_{z}\right|$ to values larger than $\approx 0.05 \mathrm{G}$ (depending on pump intensity, see below), the pattern symmetry changes to hexagonal, with $\sigma^{+}$light forming hexagons (honeycombs) and $\sigma^{-}$light forming honeycombs (hexagons), for positive (negative) $B_{z}$ values. The modulation depths of the channels are now unequal, with positive (negative) $\sigma$ being more modulated for positive (negative) $B_{z}$. For all three symmetries, the regions of excess $\sigma$ light are complementary, which leads to the conclusion that the main driver of instability is the intensity difference of the $\sigma$ components. This is seen in the subtracted NF images shown in the lowest row of Fig. 2(a) and will motivate the approximations used in Sec. VI. The inversion symmetry of $\sigma^{ \pm}$modulation amplitudes is present within experimental uncertainties for $\left|B_{z}\right|<0.05 \mathrm{G}$ and absent for higher $\left|B_{z}\right|$. To first order in spin modulation, the difference in these modulation amplitudes gives an indication of the atomic spin modulation [see Eq. (3) and the corresponding discussion in Sec. V below]. The last row of Fig. 2(a) shows antiferromagnetic states around $B_{z}=0$ and ferromagnetic states at $B_{z}=-0.09,0.12 \mathrm{G}$, with the sign of the dominant magnetization depending on the sign of $B_{z}$. The origin of this symmetry breaking is one of the subjects of this paper. Similar symmetry breaking by an external magnetic field is known to occur in the Ising model [39-42].

The NF images at large positive $B_{z}$ are shown in Fig. 2(b). The complementarity of the $\sigma$ light distributions is still present, but the patterns become disordered. For $b_{0}=110$, a residual hexagonal symmetry is observed, along with a flipping of the $\sigma$ channels, giving honeycombs for $\sigma^{+}$and hexagons for $\sigma^{-}$at positive $B_{z}$. At $b_{0}=27$, the patterns are highly disordered and a residual symmetry is not clearly discernible. 
(a) $B_{z}=-0.09 \mathrm{G}$
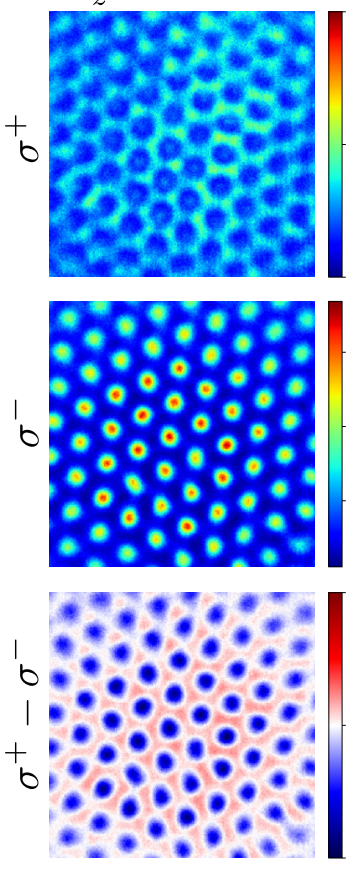
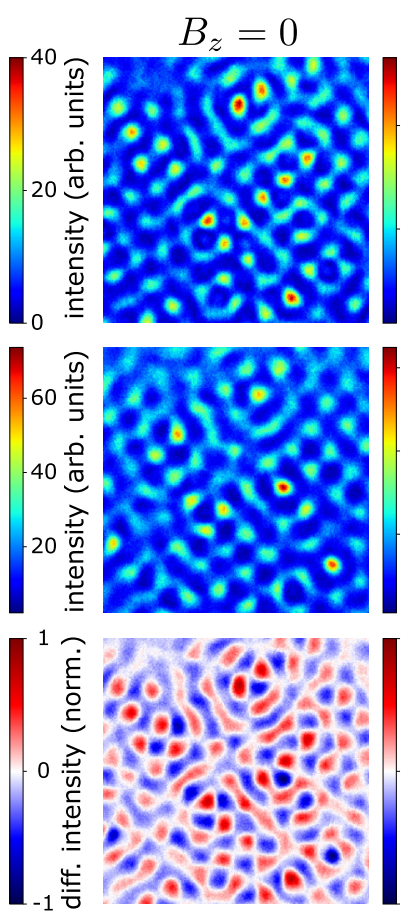
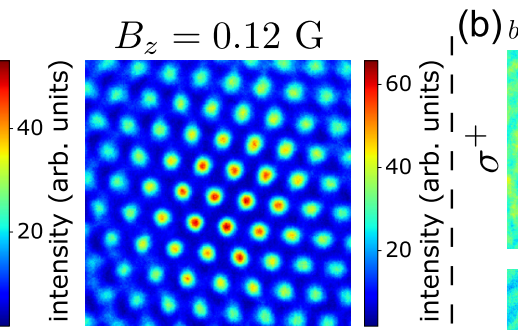

(b) $b_{0}=27, B_{z}=1.4 \mathrm{G}$
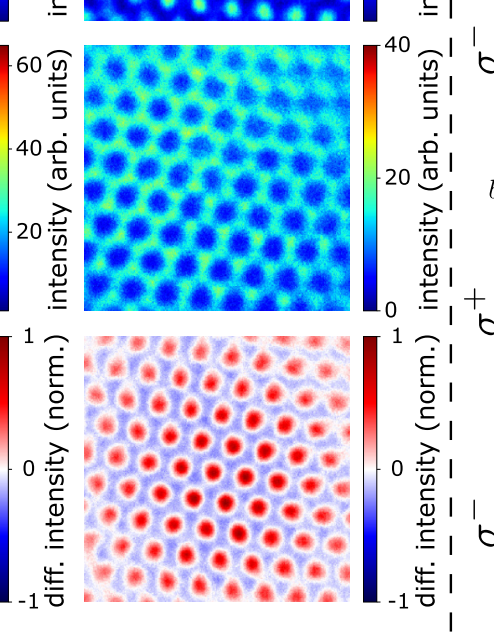
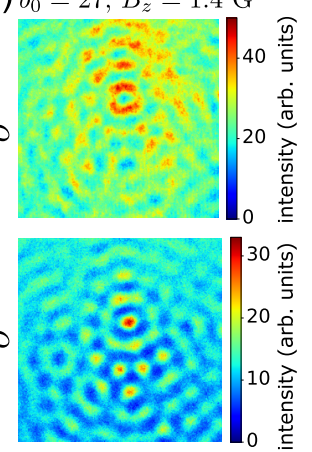

$b_{0}=110, B_{z}=0.54 \mathrm{G}$

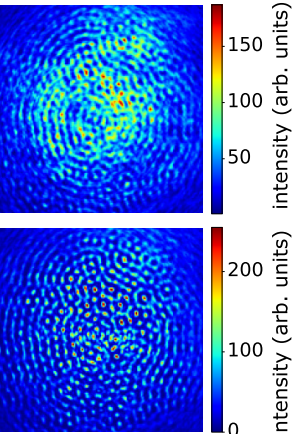

FIG. 2. Near-field images of the transmitted part of the reentrant beam intensities (see text) in the self-organized magnetization phases, for varying $B_{z}$. (a) Columns (left to right): $B_{z}=-0.09 \mathrm{G}, B_{z}=0, B_{z}=0.12 \mathrm{G}$. Rows (top to bottom): $\sigma^{+}, \sigma^{-}$and $\sigma^{+}-\sigma^{-}$(Strathclyde). The difference images are normalized to their respective maximum absolute values. The situations for $B_{z} \neq 0$ correspond to ferrimagnetic spin lattices, whereas the situation for $B_{z}=0$ corresponds to an antiferromagnetic spin lattice. (b) Top rows: $\sigma^{+}, \sigma^{-}$images for $B_{z}=1.4$ $\mathrm{G}$ (Strathclyde). Bottom rows: $\sigma^{+}, \sigma^{-}$images for $B_{z}=0.54 \mathrm{G}$ (Nice). Experimental parameters (Strathclyde): $b_{0}=27, \Delta=-14 \Gamma_{2}$, $I=10 \mathrm{~mW} / \mathrm{cm}^{2}, d=-2.9 \mathrm{~mm}, R=0.95$, field of view $0.36 \times 0.36 \mathrm{~mm}^{2}$. Experimental parameters $($ Nice $): b_{0}=110, \Delta=-24 \Gamma_{2}$, $I=22 \mathrm{~mW} / \mathrm{cm}^{2}, d=-20 \mathrm{~mm}, R=0.99$, field of view $3.15 \times 3.15 \mathrm{~mm}^{2}$.

\section{B. Diffracted power}

Figure 3 shows scans of diffracted power $P_{d}$ in the $\sigma$ polarization channels against $B_{z}$ for three values of pump

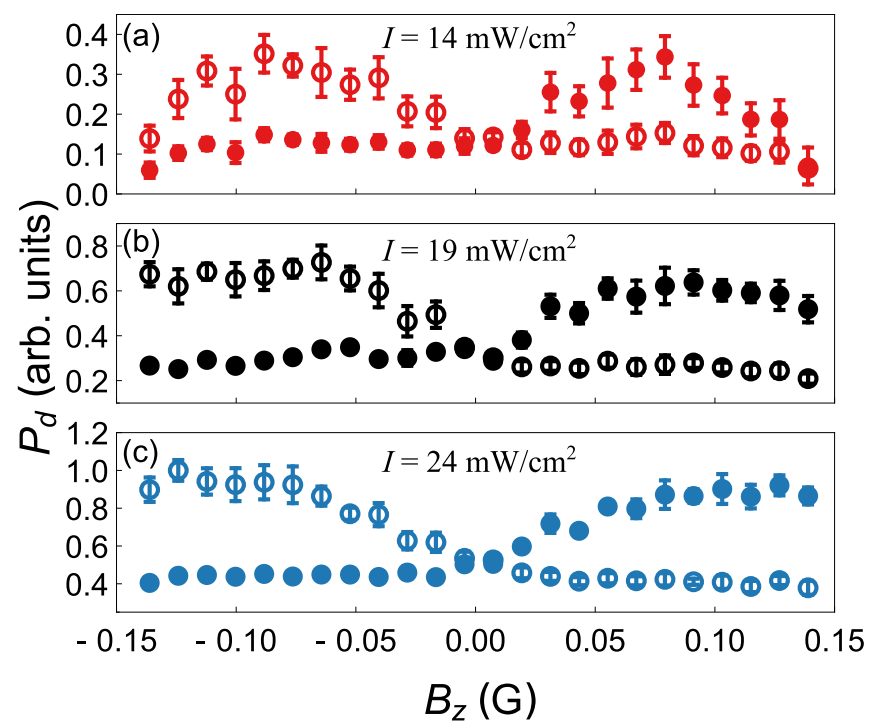

FIG. 3. Diffracted power at small longitudinal magnetic fields (Strathclyde). (a) $I=14 \mathrm{~mW} / \mathrm{cm}^{2}$. (b) $I=19 \mathrm{~mW} / \mathrm{cm}^{2}$. (c) $I=$ $24 \mathrm{~mW} / \mathrm{cm}^{2}$. Dots, $\sigma^{+}$light; circles, $\sigma^{-}$light. Experimental parameters: $\Delta=-14 \Gamma_{2}, b_{0}=27, d=-2.9 \mathrm{~mm}$. beam intensity. The $P_{d}$ was extracted from the FF data as the diffracted power in the first Talbot ring, since the power in higher rings was zero at the experimental parameters used [43]. The diffracted power in the two circular polarization channels is approximately equal near zero $B_{z}$. When we increase the $B_{z}$ magnitude above $\approx 0.03 \mathrm{G}$, the relative diffracted power in the two channels starts to differ; namely, for $B_{z}>0$ there is an increase in $\sigma^{+}$and for $B_{z}<0$ an increase in $\sigma^{-}$diffracted power. This indicates that the $\sigma^{+}\left(\sigma^{-}\right)$lattice becomes stronger for positive (negative) $B_{z}$, which is seen in NF images of Fig. 2(b).

The feature in $B_{z}$ has a subnatural linewidth; i.e., it is narrow even if it does not appear to be on the displayed span which corresponds to maximum Larmor frequencies $\Omega_{z} \approx$ $6 \times 10^{5} \mathrm{~s}^{-1} \approx 0.03 \Gamma_{2}$. Its width increases with the beam intensity, reminiscent of power broadening in the nonlinear Faraday rotation for an $F=3 \rightarrow F^{\prime}=4$ transition reported in Refs. [36,44]. We have observed this narrow feature in independent measurements of the rotation angle in a singlepass configuration at the same experimental parameters [45]. The total diffracted power has a similar qualitative behavior as the dominant polarization component and is analyzed in the next section.

\section{Threshold intensities}

The pump threshold for the magnetic transition was measured in dependence on the longitudinal field. Figure 4(a) 

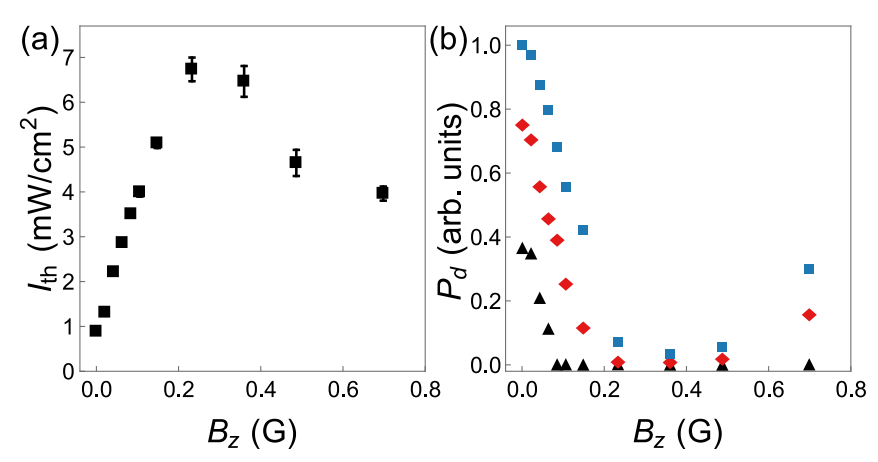

FIG. 4. Diffracted power and threshold intensity for positive $B_{z}$ (Strathclyde). (a) Threshold pump intensity. (b) Total diffracted power for varying input beam intensity. Triangles (black): $I=$ $3.1 \mathrm{~mW} / \mathrm{cm}^{2}$. Diamonds (red): $I=5.5 \mathrm{~mW} / \mathrm{cm}^{2}$. Squares (blue): $I=7.1 \mathrm{~mW} / \mathrm{cm}^{2}$. Experimental parameters: $\Delta=-14 \Gamma_{2}, b_{0}=27$, $d=1.3 \mathrm{~mm}$.

shows threshold beam intensity $I_{t h}$ against $B_{z}$. The threshold is minimal in zero field. For small $B_{z}, I_{t h}$ increases with $B_{z}$ and peaks at $\approx 0.25 \mathrm{G}$. On further increasing $B_{z}, I_{t h}$ begins to drop and levels off to a constant value. It will be shown in Secs. V and VI that this dependence on $B_{z}$ can be accounted for in a theoretical model describing the atoms as a spin-1 medium.

Scans of the total diffracted power for $B_{z}>0$ are shown in Fig. 4(b). Depending on the pump beam intensity with respect to the threshold [see Fig. 4(a)], different behaviors are observed. At low beam intensities above threshold, the $P_{d}$ drops at small $B_{z}$ and remains zero for higher field magnitudes. As we increase the beam intensity, the $P_{d}$ feature in $B_{z}$ gets broader and exhibits a revival after an initial strong decrease. This is related to the results of Fig. 4(a), as the increase (decrease) of the pattern threshold corresponds to a decrease (increase) of diffracted power at a fixed pump intensity. Input intensities below $4 \mathrm{~mW} / \mathrm{cm}^{2}$ are below the minimum threshold for the revival at high- $B_{z}$ fields and the magnetic ordering occurs only in the central lobe. The width of the lobe increases with intensity, indicating power broadening of a magneto-optical resonance. The total diffractive power and the dominant polarization component show qualitatively similar behavior.

\section{THEORETICAL MODEL}

\section{A. Atom dynamics}

We now outline the theoretical model for the internal degrees of freedom of the atomic medium interacting with the pump laser. The large pump beam detuning in our experiments allows for the use of the low saturation approximation, where atom-light interaction is modeled by considering only the ground-state populations and coherences of the density matrix $\rho$; see, e.g., Refs. [27,35]. In addition to this, for simplicity we also approximate the experimentally excited $F=2 \rightarrow F^{\prime}=$ 3 transition of the $\mathrm{D} 2$ line of ${ }^{87} \mathrm{Rb}$ with an $F=1 \rightarrow F^{\prime}=2$ transition as it contains the relevant multipoles. It should be also noted that the multipoles of rank higher than 2 do not provide a feedback to the light field. Choosing the quantization axis parallel to the pump propagation direction $z^{\prime}$ and setting the transverse magnetic fields to zero, we identify the relevant

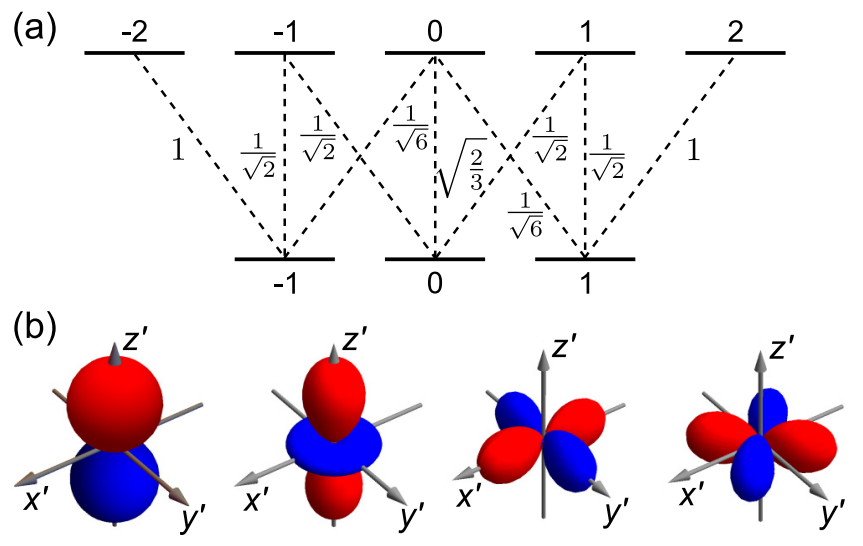

FIG. 5. Model atomic system. (a) Zeeman sublevel structure of the $F=1 \rightarrow F^{\prime}=2$ transition with the corresponding ClebschGordan coefficients. (b) Illustration of symmetries of the tensors related to the magnetic moments $w, x, u, v$, from left to right.

atomic variables to be $u=2 \operatorname{Re}\left(\rho_{1-1}\right), v=2 \operatorname{Im}\left(\rho_{1-1}\right), w=$ $\rho_{11}-\rho_{-1-1}$, and $x=\rho_{11}+\rho_{-1-1}-2 \rho_{00}$ (where $\rho_{i, j}$ are the density matrix elements of the $i$ th and $j$ th Zeeman sublevels of the spin- 1 ground state), given respectively by the expectation values: $\left\langle F_{x}^{2}-F_{y}^{2}\right\rangle,\left\langle F_{x} F_{y}+F_{y} F_{x}\right\rangle,\left\langle F_{z}\right\rangle$, and $\left\langle 3 F_{z}^{2}-F^{2}\right\rangle$, where $F^{2}$ and $F_{x, y, z}$ are hyperfine angular momentum operators [46]. Each of the above variables is also proportional to a coefficient of the irreducible tensor expansion of the density matrix, known as a polarization moment, the knowledge of which is sufficient to describe the angular momentum state of an atomic ensemble [46]. The characteristic spatial symmetry of each tensor is given by the corresponding spherical harmonic function, as shown in Fig. 5. The $w$ variable is also called orientation (spin) and corresponds to a dipole, whereas the alignment $x$ and coherences $u$ and $v$ correspond to quadrupoles.

Temporal dynamics of the atomic variables is described by a set of optical Bloch equations. After adiabatic elimination of the optical coherences and the excited state variables (up to the order of $\Omega_{ \pm} / \Delta$ [35], where $\Omega_{ \pm}$are Rabi frequencies of the $\sigma$ light components), one is left with a set of four equations for the ground-state variables $[25,26,45]$

$$
\begin{aligned}
\dot{u}= & -\Gamma_{c} u+\left(2 \Omega_{z}+\frac{5}{6} \mathcal{D} \bar{\Delta}\right) v+\frac{1}{6} P_{\Lambda-} \bar{\Delta} w \\
& -\frac{1}{9} P_{\Lambda+} x+\frac{5}{18} P_{\Lambda+}, \\
\dot{v}= & -\Gamma_{c} v-\left(2 \Omega_{z}+\frac{5}{6} \mathcal{D} \bar{\Delta}\right) u+\frac{1}{6} P_{\Lambda+} \bar{\Delta} w \\
& +\frac{1}{9} P_{\Lambda-} x-\frac{5}{18} P_{\Lambda-}, \\
\dot{w}= & -\Gamma_{w} w-\frac{1}{6} P_{\Lambda-} \bar{\Delta} u-\frac{1}{6} P_{\Lambda+} \bar{\Delta} v-\frac{1}{9} \mathcal{D} x+\frac{5}{18} \mathcal{D}, \\
\dot{x}= & -\Gamma_{x} x-\frac{1}{3} P_{\Lambda+} u+\frac{1}{3} P_{\Lambda-} v+\frac{1}{3} \mathcal{D} w+\frac{5}{18} \mathcal{S},
\end{aligned}
$$


where the pump rates are

$$
\begin{aligned}
\mathcal{S} & =\frac{1}{\Gamma_{2}}\left[\frac{\left|\Omega_{+}\right|^{2}}{1+\left(\bar{\Delta}-\bar{\Omega}_{z}\right)^{2}}+\frac{\left|\Omega_{-}\right|^{2}}{1+\left(\bar{\Delta}+\bar{\Omega}_{z}\right)^{2}}\right], \\
\mathcal{D} & =\frac{1}{\Gamma_{2}}\left[\frac{\left|\Omega_{+}\right|^{2}}{1+\left(\bar{\Delta}-\bar{\Omega}_{z}\right)^{2}}-\frac{\left|\Omega_{-}\right|^{2}}{1+\left(\bar{\Delta}+\bar{\Omega}_{z}\right)^{2}}\right], \\
P_{\Lambda+} & =\frac{2}{\Gamma_{2}} \frac{\operatorname{Re}\left(\Omega_{+}^{*} \Omega_{-}\right)}{1+\bar{\Delta}^{2}}, P_{\Lambda-}=\frac{-2}{\Gamma_{2}} \frac{\operatorname{Im}\left(\Omega_{+}^{*} \Omega_{-}\right)}{1+\bar{\Delta}^{2}},
\end{aligned}
$$

and the decay rates are $\Gamma_{w}=\gamma+\frac{1}{6} \mathcal{S}, \Gamma_{x}=\gamma+$ $\frac{11}{18} \mathcal{S}$, and $\Gamma_{c}=\gamma+\frac{5}{6} \mathcal{S}$. Here, $\gamma$ is an effective decay rate of the Zeeman ground-state populations and coherences, detuning is normalized as $\bar{\Delta}=\Delta / \Gamma_{2}$, and $\Omega_{z}$ is the Larmor precession frequency in a longitudinal magnetic field. Rabi frequencies $\Omega_{ \pm}$are related to the electric fields $E_{ \pm}$as $\Omega_{ \pm}=\mu_{d} E_{ \pm} / \hbar$, where $\mu_{d}$ is the dipole matrix element of the stretched state optical transitions. In the following, we use the expression $\Omega_{ \pm}=\Gamma_{1} \sqrt{I_{ \pm} / 2 I_{s}}$, with $I_{s}=1.669 \mathrm{~mW} / \mathrm{cm}^{2}$. Equations (1) are valid in the case when no transverse magnetic fields are present, and the $|\Delta m|=1$ ground-state coherences vanish. When we neglect the coupling to other multipoles, Eqs. (1) show that the orientation $w$ is driven by an intensity difference in the two circular polarization components, the $x$ variable is driven by light polarized along any direction, whereas $u$ and $v$ couple most strongly to light polarized along $x^{\prime}$ or $y^{\prime}$ and at $45^{\circ}$ to $x^{\prime}$ or $y^{\prime}$, respectively. The pumping of $w$ and $x$ is related to incoherent processes (i.e., insensitive to the phase between the $\sigma^{+}$and $\sigma^{-}$components), whereas $u$ and $v$ are pumped in a coherent way (i.e., sensitive to the relative light phase of the circular components).

\section{B. Field evolution}

We will consider the propagation of the slowly varying electric field envelopes $\tilde{E}_{ \pm}=\tilde{F}_{ \pm}+\tilde{B}_{ \pm}$, where $\tilde{F}_{ \pm}$are the forward- and $\tilde{B}_{ \pm}$the backward-propagating $\sigma$ electric field components. In the next two subsections, we normalize the electric fields as $E_{0}=\Omega_{0} / \sqrt{\Gamma_{2}\left(1+\bar{\Delta}^{2}\right)}$, where $\Omega_{0}=\Omega_{+}=$ $\Omega_{-}$since we use a beam polarized along the $y^{\prime}$ direction, and we write $I_{0}=\left|E_{0}\right|^{2}$ for the intensity of each circular component.

The pump terms in Eqs. (1) are all quadratic in the optical fields and will include terms $\approx e^{ \pm 2 i k z}$ due to the interference of the counterpropagating beams. As the groundstate dynamics is rather slow, the atoms will traverse several optical wavelengths on the timescale of the state dynamics $(\gtrsim 10 \mu \mathrm{s})$, and so these grating terms will be averaged out and will not contribute to the response of the atomic variables. We will therefore ignore these "longitudinal grating" terms, replacing the pump terms $\mathcal{S}, \mathcal{D}, P_{\Lambda_{+}}$, and $P_{\Lambda_{-}}$ with their spatial averages $\kappa_{+}\left(\left|\tilde{F}_{+}\right|^{2}+\left|\tilde{B}_{+}\right|^{2}\right)+\kappa_{-}\left(\left|\tilde{F}_{-}\right|^{2}+\right.$ $\left.\left|\tilde{B}_{-}\right|^{2}\right), \kappa_{+}\left(\left|\tilde{F}_{+}\right|^{2}+\left|\tilde{B}_{+}\right|^{2}\right)-\kappa_{-}\left(\left|\tilde{F}_{-}\right|^{2}+\left|\tilde{B}_{-}\right|^{2}\right), 2 \operatorname{Re}\left(\tilde{F}_{+}^{*} \tilde{F}_{-}+\right.$ $\left.\tilde{B}_{+}^{*} \tilde{B}_{-}\right)$, and $-2 \operatorname{Im}\left(\tilde{F}_{+}^{*} \tilde{F}_{-}+\tilde{B}_{+}^{*} \tilde{B}_{-}\right)$, respectively, where $\kappa_{ \pm}=\left(1+\bar{\Delta}^{2}\right) /\left[1+\left(\bar{\Delta} \mp \bar{\Omega}_{z}\right)^{2}\right]$, and $\bar{\Omega}_{z}=\Omega_{z} / \Gamma_{2}=0.23 \times$ $B_{z} / \mathrm{G}$, where we have used $g_{F}=0.5$ (taken from the $F=2$ experimental ground state) for the Landé $g$ factor. The coefficients $\kappa_{ \pm}$take into account the influence of Zeeman detuning on pumping by $\sigma$ light components. This dependence in the $\mathcal{D}$ pump rate gives rise to the incoherent part of the nonlinear
Faraday effect. The coherent pump terms $P_{\Lambda \pm}$ do not contain the dependence on $B_{z}$ as the coherences vanish at small $\Omega_{z} \lesssim$ $\Delta$, and the approximation is justified in more detail in the supplementary material of Ref. [26].

We take no further account of atomic motion and assume that the atomic variables respond only to the local optical fields. This is justified as the cold atoms traverse only a fraction of the transverse pattern period during the onset of pattern formation. The field evolution equations for the forward-propagating fields are now

$$
\frac{\partial}{\partial z^{\prime}} \tilde{F}_{ \pm}=i \frac{\phi_{ \pm}^{0}}{L}\left[\left(1 \pm \frac{3}{4} w+\frac{1}{20} x\right) \tilde{F}_{ \pm}+\frac{3}{20}(u \mp i v) \tilde{F}_{\mp}\right],
$$

where $L$ is the longitudinal length of the atomic cloud, $\phi_{ \pm}^{0}=$ $\frac{b_{0}}{2} \frac{\left(\bar{\Delta} \mp \bar{\Omega}_{z}\right)}{1+(\bar{\Delta} \mp \bar{\Omega})^{2}}$ is the linear phase shift, including the linear Faraday effect, and in analytical calculations we neglect the imaginary part of the dielectric susceptibility (i.e., absorption) as we are in a regime where $|\Delta| \gg \Gamma_{2}$. Equations (3) elucidate the optical nonlinearities at work in the atomic medium. The constant term is linear refraction. The $w$ and $x$ terms are due to stronger light coupling to atoms pumped into stretched states. The $w$ term describes the action of an orientation, i.e., of a dipole state with unequal occupation in the opposite spin states, and acts oppositely on the two circular polarization components. It provides circular birefringence, leading to a self-focusing nonlinearity similar to the one occurring in the spin-1/2 system $[29,30]$, with the difference here being that pumping drives populations into bright instead of dark states. The alignment term $x$ is a consequence of stronger coupling of both $\sigma$ light components to populations in either of the two stretched states and is not polarization selective. The $u, v$ terms are due to coherent cross coupling between the two circular light polarization modes via a shared excited state in a $\Lambda$ subsystem and allow for generation of circular light components of opposite polarizations than the input [47].

The transverse coupling of the atoms is provided by free space diffraction during propagation from the end of the medium and back, which is governed by

$$
\frac{\partial}{\partial z^{\prime}} \tilde{F}_{ \pm}=-\frac{i}{2 k} \Delta_{\perp} \tilde{F}_{ \pm}
$$

where $\Delta_{\perp}$ is the transverse Laplacian. Integration of this equation yields the backward fields $\tilde{B}_{ \pm}$.

\section{NUMERICAL RESULTS}

We have solved numerically equations (1) in both 1D and 2D geometries. The incident linearly polarized beam propagates through the medium with the optical response given by (3) with both absorption and dispersion included. The atomic cloud is modeled as a thin slab with 128 (in $2 \mathrm{D}$ : $128 \times 128$ ) grid points with dynamics described by Eqs. (1). The spatial coupling of the atoms is provided by free space diffraction between the end of the medium and the mirror. For this, Eq. (4) is solved in Fourier space.

The threshold of pattern formation is characterized by an exponential growth of the laser beam profile modulation, caused by a corresponding growth in the modulation of the 

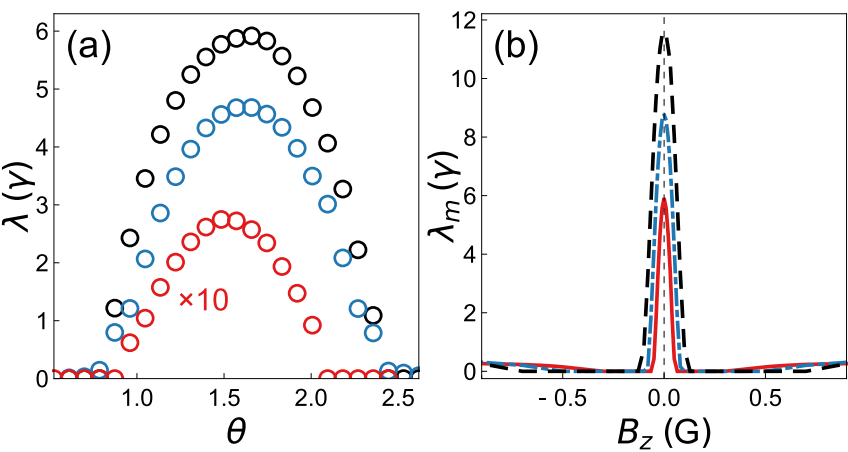

FIG. 6. Growth rate of the patterns for a one-dimensional (1D) numerical simulations (see text). (a) Growth rate $\lambda$ at $I_{0}=$ $10 \mathrm{~mW} / \mathrm{cm}^{2}$ against the diffractive phase shift $\theta$ (see text) for $B_{z}=0$ (black, top circles), $B_{z}=20 \mathrm{mG}$ (blue, middle circles), and $B_{z}=1.5 \mathrm{G}$ (red, bottom circles). The values for $B_{z}=1.5 \mathrm{G}$ were multiplied by 10 for visibility. (b) Scan of the fastest growth rate $\lambda_{m}$ against $B_{z}$ for three input intensities $I_{0}: 10 I_{s}$ (red, solid), $15 I_{s}$ (blue, dot-dashed), and 20I (black, dashed). Parameters: $b_{0}=30$, $\Delta=-10 \Gamma_{2}, R=0.95, \gamma=10^{-4} \Gamma_{2}$.

medium variable driving the self-organization. In our case, this is mainly the spin $w$ variable (see Sec. VI). The initial spontaneously appearing seed perturbation with the largest growth rate $\lambda$ will evolve into a steady-state solution seen in the experiment [37]. The analysis of scans of $\lambda$ against diffractive phase shift $\theta=q^{2} d / k$ (where $q$ is the transverse wave number) at three different $B_{z}$ 's shown in Fig. 6(a) leads to the conclusion that the fastest growing patterns have a diffractive phase shift of approximately $\pi / 2$. A critical diffractive phase shift of $\pi / 2$ appears for instabilities in a spin-1/2 system and a Kerr slab [20,29]. At $b_{0}=30$ and for input intensities used in Fig. 6(b), the maximal growth rate $\lambda_{m}$ initially drops to zero, and then exhibits a recurrence for larger $\left|B_{z}\right|$, as shown in Fig. 6(b). The width of the central features increases for a larger pump intensity, due to power broadening, visible also in experimental measurements of diffracted power.

The NF images of simulated 2D patterns at three different $B_{z}$ values are plotted in Fig. 7. At zero $B_{z}$, the patterns exhibit a square symmetry with stripelike defects. This is similar to the experimental pattern realizations, where stripelike defects are also observed. The modulation depths of the $\sigma$ beam profiles are equal, with their difference giving a lattice with neighboring sites of equal magnitude and opposite helicities. The corresponding profile of the spin $w$ variable mimics this behavior, with neighboring atomic spins alternating between up and down directions with equal maximal magnitudes. This constitutes an antiferromagnetic spin state. Observing the NF profiles of the $\sigma$ polarization channels can thus reveal the underlying spin structure inside the atomic medium and justifies the approach taken in Fig. 2 to infer the magnetic distribution in the medium from the difference of the NF images of the circular components. For small $B_{z}$ 's, the inversion symmetry of the system is broken and patterns with hexagonal symmetry appear. For negative (positive) $B_{z}$, the $\sigma^{+}$patterns are honeycombs (hexagons) and the $\sigma^{-}$patterns are hexagons (honeycombs), as in the experiment. The subtracted $\sigma$ intensity profiles and the $w$ variable both show

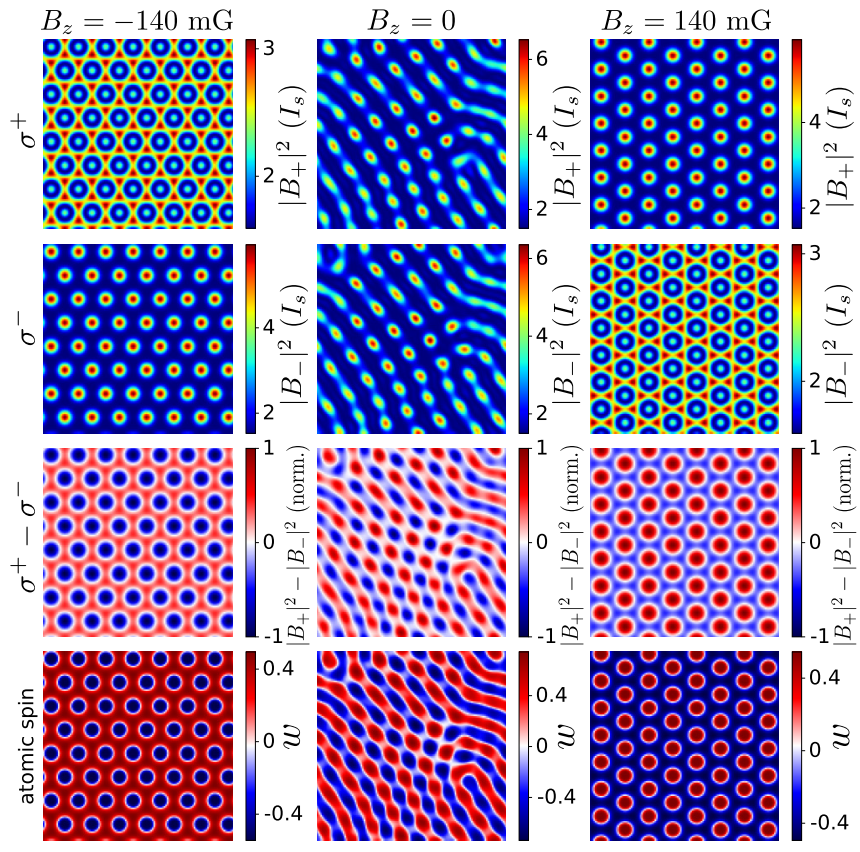

FIG. 7. Near-field steady-state data (depicting intensity of the reentrant feedback field $B_{ \pm}=\tilde{B}_{ \pm}(z=L)$ and atomic orientation $w$ ), taken from two-dimensional (2D) simulations. Columns (left to right): $B_{z}=-140 \mathrm{mG}, B_{z}=0, B_{z}=140 \mathrm{mG}$. Rows (top to bottom): $\left|B_{+}\right|^{2},\left|B_{-}\right|^{2},\left|B_{+}\right|^{2}-\left|B_{-}\right|^{2}$ and the $w$ variable. Simulation parameters: $b_{0}=60, I_{0}=15 I_{s}, \Delta=-10 \Gamma_{2}, R=0.95, \gamma=10^{-4} \Gamma_{2}$, simulation time: $2 \times 10^{4} / \Gamma_{2}$. The size of the numerical grid was adjusted to contain seven periods of the lattice. Periodic boundary conditions are used in the simulations.

positive (negative) hexagons for positive (negative) $B_{z}$. The modulation depth of the positive (negative) spin sublattice is greater at positive (negative) $B_{z}$, resulting in a net positive (negative) magnetization. This constitutes a ferromagnetic spin state.

\section{ANALYTICAL CALCULATIONS}

The state of the spin-1 system analyzed is determined by four coupled dynamical equations (1) for the variables $u, v, w, x$, evolving on similar timescales. This situation is quite unlike most previous work in the single feedback mirror (SFM) configuration, where time-dependent perturbation analysis is done by considering the perturbation evolution of a single (slow) degree of freedom of the optically nonlinear medium, e.g., atomic density [5,6] or spin [29,30] in atomic media, charge carrier density in direct band-gap semiconductors [48], or the phase difference between ordinary and extraordinary waves in liquid crystal valves [49]. In contrast to the numerical results of Sec. V, in this section we present analytical results for a simplified model, taking into account only the perturbations and feedback to the spin- $w$ variable. This is motivated by the numerical simulations indicating the dominance of the orientation in the magnetic ordering. Although only approximate, this model is illustrative as it provides physical explanations for the variation of thresholds and symmetries at small $B_{z}$, consistent with both experimental and numerical results. We will compare the results of this 
model with those for a further simplified $w$-only model, which is effectively a spin- $1 / 2$ model, derived by putting $u=v=$ $x=0$ in (1) and (3), and demonstrate the inadequacy of the latter for describing the pattern properties.

\section{A. Linear stability analysis}

We now calculate the threshold intensity of the pattern formation in the spin-1 model. In writing Eqs. (3), we have made use of the thin-medium approximation, in which the cloud is diffractively thin and the patterns form due to interference of the fields $F_{ \pm}+B_{ \pm}$at the end of the medium, where $F_{ \pm}=F_{ \pm}^{0}=E_{0}$ and $B_{ \pm}=\tilde{B}_{ \pm}(z=L)[50]$. This is justified as the medium is sufficiently optically thin, and we use mirror positions just outside the end of the medium, so that the diffraction and the nonlinear phase shift within the medium are not the dominant effects but not so far from it to observe competition with the higher order Talbot modes [43].

The backward-propagating field re-entering the medium is of the form

$$
B_{ \pm}=B_{ \pm}^{0}\left(1+b_{ \pm}\right),
$$

where $B_{ \pm}^{0}=\sqrt{R} E_{0}$ is the homogeneous part of the backwardpropagating field, $R$ is the mirror reflectivity, and $b_{ \pm}$are small perturbations in the field caused by a transverse perturbation in the atomic spin of the form $\delta w \cos \left(q x^{\prime}\right)$. We relate the spin perturbation with $b_{ \pm}$by using $w=w_{h}+\delta w \cos \left(q x^{\prime}\right)$ in Eq. (3) (with $u=v=x=0$ ), where $w_{h}$ is the homogeneous part of the spin, and integrating Eq. (4) to the mirror and back:

$$
b_{ \pm}= \pm i \frac{3}{4} \phi_{ \pm}^{0} e^{i \theta} \delta w \cos \left(q x^{\prime}\right),
$$

where $d$ is the mirror distance from the end of the medium. Homogeneous values of the atomic variables are calculated from (1) using $F_{ \pm}^{0}+B_{ \pm}^{0}$ for the electric fields. In writing the relation (6), we have neglected the influence of the perturbations in the higher order magnetic multipoles, as motivated by experimental and numerical results of the full model which indicate that the $w$ variable is the main driver of instability.

Equations (5) and (6) illustrate how the pattern formation occurs. The plane wave enters the cloud and acquires a transverse phase modulation from the atomic spins inside the medium. As we work in the thin medium approximation, we neglect the phase modulation in the forward-propagating beam at the end of the cloud, since the structured feedback by the backward-propagating beam is expected to dominate the pattern formation. In linear stability analysis (LSA), we look at the growth of an initial cosine spin perturbation with a given transverse wave number $q$. The diffraction of the phase modulated beam from the end of the cloud and back causes the transverse profile to continually vary along $z^{\prime}$, interchanging planes of phase and amplitude modulation, due to the Talbot effect [51], parametrized in our model via $\theta$. Since $\theta$ depends on $q$, for a given transverse perturbation there is a certain $b_{ \pm}$ at a given mirror position $d$. As is shown in Fig. 6(a), the fastest growing perturbations will occur for a certain critical $\theta$, and this is in general the $\theta$ value seen in the steadystate patterns observed experimentally [37]. We will here use the critical phase shift of $\theta=\pi / 2$, meaning the instability maximizes modulation in the difference pump rate $\mathcal{D}$, as is consistent with both experimental and numerical data of the full model. The patterns thus grow from initial noise due to the feedback provided by $b_{ \pm}$, via the birefringent nonlinearity given by the $\delta w$ term, effectively inducing interatomic interactions mediated by the light field in this out-of-equilibrium system.

We are now interested in solutions corresponding to transverse patterns, the dynamics of which is characterized by exponential growth $\delta w \sim e^{\lambda t}$ (where $\lambda \in \mathbb{R}$ ) near threshold. Inserting this form of $\delta w$ into the dynamical equation for $w$, we get for $\lambda$ :

$$
\begin{aligned}
\lambda= & -\left[\gamma+\frac{I_{0} \bar{\kappa}}{6}(1+R)\right]+\frac{I_{0} w_{h} R}{4}\left(\kappa_{+} \phi_{+}^{0}-\kappa_{-} \phi_{-}^{0}\right) \\
& +\frac{\bar{\Delta} v_{h} I_{0} R}{4}\left(\phi_{+}^{0}-\phi_{-}^{0}\right)+\frac{I_{0} R}{12}\left(2 x_{h}-5\right)\left(\kappa_{+} \phi_{+}^{0}+\kappa_{-} \phi_{-}^{0}\right),
\end{aligned}
$$

where $u_{h}, v_{h}, w_{h}, x_{h}$ are the homogeneous parts of the $u, v, w, x$ variables and $\bar{\kappa}=\kappa_{+}+\kappa_{-}$. Setting now $\lambda=0$, we obtain the expression for total threshold intensity $I_{t h}$ of the linearly polarized input pump beam:

$$
\begin{aligned}
I_{t h}= & 2 \gamma\left[-\frac{\bar{\kappa}}{6}(1+R)+\frac{w_{h}^{t h} R}{4}\left(\kappa_{+} \phi_{+}^{0}-\kappa_{-} \phi_{-}^{0}\right)\right. \\
& \left.+\frac{\bar{\Delta} v_{h}^{t h} R}{4}\left(\phi_{+}^{0}-\phi_{-}^{0}\right)+\frac{R}{12}\left(2 x_{h}^{t h}-5\right)\left(\kappa_{+} \phi_{+}^{0}+\kappa_{-} \phi_{-}^{0}\right)\right]^{-1},
\end{aligned}
$$

where the subscript "h" and superscript "th" denominate the homogeneous and threshold parameter values, respectively. The solution (8) is inserted into Eqs. (1) to get the threshold values of the homogeneous atomic variables, which allows us to calculate the $I_{t h}$.

The scan of $I_{t h}$ (in $\mathrm{mW} / \mathrm{cm}^{2}$ ) against $B_{z}$ is plotted in Fig. 8 for three different solutions: the full solution using (8), the spin-1/2 model introduced before (i.e., keeping $x=$ $u=v=0$ throughout and not only in the feedback terms, but keeping the incoherent linear and nonlinear Faraday effect), and a simplified solution keeping $x, u, v$ in the homogeneous terms but neglecting both the linear and incoherent nonlinear Faraday effects [25], i.e., setting $\kappa_{ \pm}=1, \phi^{0}=\phi_{ \pm}^{0}$ :

$$
I_{t h} \approx \frac{2 \gamma}{-\frac{1}{3}(1+R)+\frac{R \phi^{0}}{6}\left(2 x_{h}^{t h}-5\right)} .
$$

It should be noted that $\phi^{0}<0$ for the red detuning condition under study. The full solution (red line) has a minimum threshold for $B_{z}=0$. It increases with incresing $\left|B_{z}\right|$. This qualitatively mimics the small $\left|B_{z}\right|$ experimental results at $b_{0}=27$. We note that we have used $b_{0}=30$ in our calculations, as the experimental values have an estimated uncertainty of $\lesssim 5$, and the chosen value gives a more robust agreement with experiment. At small $\left|B_{z}\right|$, the behavior is well reproduced by Eq. (9) (see dash-dotted green line), where the $B_{z}$ dependence arises solely due to $x_{h}^{t h}$ (see Fig. 9). The origin of this dependence is explained below. At larger $\left|B_{z}\right|$, the full solution and the solution (9) start to deviate with the threshold of the full solution rising, whereas the solution (9) saturates to a finite value. 


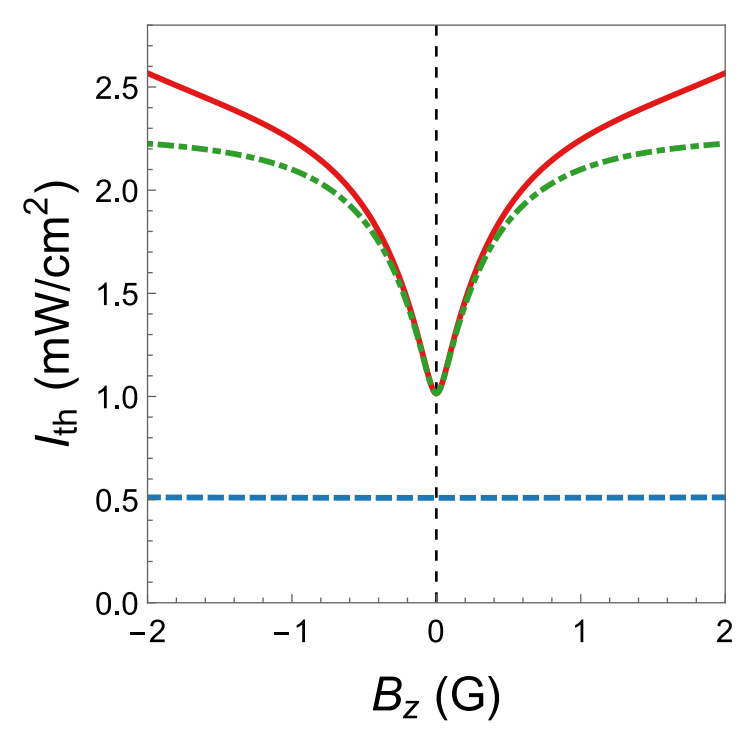

FIG. 8. Variation of threshold intensity with $B_{z}$. Red solid curve: full solution using (8). Green dot-dashed curve: solution for the model neglecting both linear and nonlinear Faraday effects [using (9)]. Blue dashed curve: solution of $w$-only model (see text). Parameters: $\Delta=-14 \Gamma_{2}, R=0.95, b_{0}=30, \gamma=3 \times 10^{-4} \Gamma_{2}$.

The onset of pattern formation happens at the intensity for which modulation depumping [first term in Eq. (7) stemming from the sum pump rate $\mathcal{S}$ term in the relaxation terms of Eqs. (1)] is equal to pumping due to the intensity modulated pump rate [last term of Eq. (7) stemming from the difference pump rate $\mathcal{D}$ term]. The $x_{h}$ dependence arises from the term $\propto-\mathcal{D} x$ in the third equation of (1). Writing $x$ as $x=$
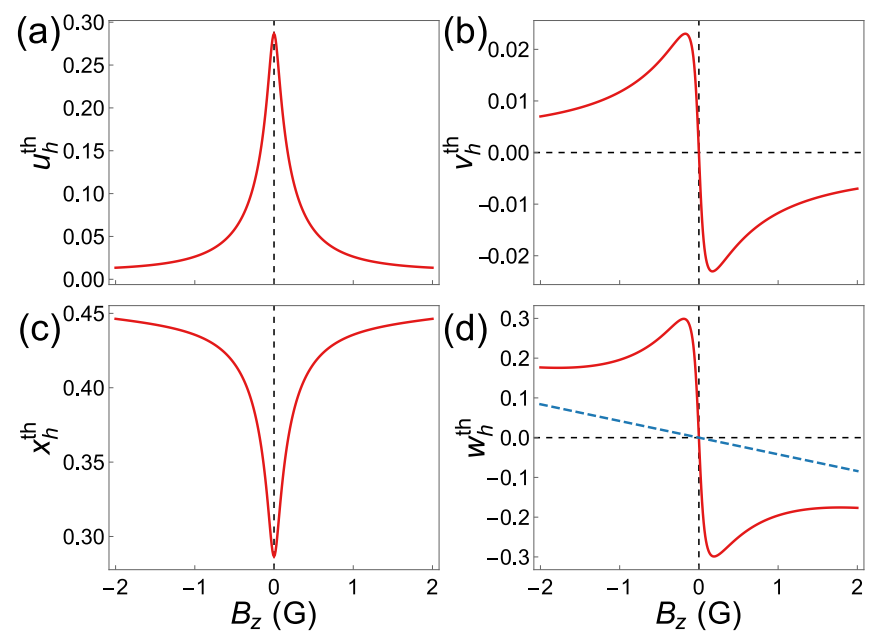

FIG. 9. Homogeneous solutions of (1) at threshold, against $B_{z}$. Coherences (a) $u$ and (b) $v$, (c) alignment $x$ and (d) orientation $w$. The narrow features for small $B_{z}$ are caused by magnetic field coupling to the coherences $u$ and $v$ (i.e., coherent nonlinear Faraday rotation), whereas the features at large $B_{z}$ are caused by linear and nonlinear Faraday rotation due to detuning via Zeeman shifts (see text). This is confirmed by the solution of the $w$-only model, represented by the blue dash-dotted curve of panel (d). The scan is representative of the single-pass behavior for the $F=1 \rightarrow F^{\prime}=2$ transition at low pump saturation. Parameter values are as in Fig. 8.
$1-3 \rho_{00}$ (using $\rho_{-1-1}+\rho_{00}+\rho_{11}=1$ ), this term becomes $-\mathcal{D} x=\mathcal{D}\left(3 \rho_{00}-1\right)$. Thus, for $\mathcal{D}>0(\mathcal{D}<0)$ an increase of population in the $m=0$ state will increase the effective pumping rate of the spin into the $m=1(m=-1)$ state. The origin of the variation of $x_{h}^{\text {th }}$ at small $\left|B_{z}\right|$ is in the coupling term $-\frac{1}{3} P_{\Lambda+} u$ of the fourth equation in (1), the details of which will be explained at the end of this section. In short, Fig. 9 shows that the coherent nonlinear Faraday effect creates a magneto-optical resonance for the coherences $u, v$. The resonance in $u$ couples to $x$, which reduces (increases) the spin modulation pump rate and thus increases (reduces) the threshold, for larger (smaller) $x$ [see Eq. (9)]. The resonance in $v$ couples to the $w$ and causes it to rotate in $B_{z}$, which leads to the deviation of the full and w-only solutions in Fig. 9(d), as explained at the end of this section.

The dashed curve in Fig. 8 is for the spin-1/2,w-only, model, which includes both linear and incoherent nonlinear Faraday effects. It does not show a $B_{z}$ dependence. The large difference between this and the solid threshold curve at all $B_{z}$ shows that the $w$-only model cannot account for the $B_{z}$ dependence of the experimental threshold intensity presented in Fig. 4(b). This demonstrates that the system at hand is more complex and potentially more rich than the previously studied spin-1/2 model of Refs. [29,30]. Threshold curves of the instability versus $B_{z}$ for a spin-1/2 system presented in Ref. [52] were all obtained in small transverse magnetic fields and their extrapolation to zero transverse field is compatible with a flat threshold curve versus $B_{z}$.

The decrease of threshold with large $B_{z}$ seen in experiments is not reproduced by any of these models at these parameter values, which leads us to the conclusion that the perturbations in the higher order magnetic multipoles are responsible for a threshold decrease at large $B_{z}$, implied by the results in Fig. 6(b).

\section{B. Inversion symmetry breaking}

To calculate the symmetries of patterns at threshold, we employ the method of nonlinear analysis (NLA) used by D'Alessandro and Firth for SFM patterns in a Kerr medium [48]. We will reformulate our problem as a single partial differential equation of infinite order, describing the temporal evolution of spin perturbation $\delta \tilde{w}$, and end up with a set of Ginzburg-Landau equations for the roll state amplitudes, from which we calculate the variation of the allowed pattern symmetries with $B_{z}$.

The backward-propagating fields $B_{ \pm}$reflected from the mirror and re-entering the medium can be related to the fields exiting the medium by formally integrating the free space diffraction equation to the mirror and back, giving

$$
B_{ \pm}=\sqrt{R} E_{0} e^{-i \sigma \Delta_{\perp}} e^{ \pm i \frac{3 \phi_{ \pm}^{0}}{4} \delta \tilde{w}}
$$

where $\sigma=d / k$ and we use an ansatz for the modulation $\delta \tilde{w}$ :

$\delta \tilde{w}=\varepsilon\left(A_{1}(t) e^{i \mathbf{q}_{1} \cdot \mathbf{r}}+A_{2}(t) e^{i \mathbf{q}_{2} \cdot \mathbf{r}}+A_{3}(t) e^{i \mathbf{q}_{3} \cdot \mathbf{r}}+\right.$ c.c. $) / 2$,

where $\varepsilon$ is a bookkeeping parameter. This solution corresponds to a superposition of three roll states with wave vectors $\mathbf{q}_{i}$ (with $i=1,2,3$ ) and is a reasonable assumption for pump 
intensities near threshold. For the input pump intensities of the $\sigma$ components, we will thus use $\left|F_{+}\right|^{2}=\left|F_{-}\right|^{2}=I_{0}=p I_{t h} / 2$, where the parameter $p$ is close to 1 .
Inserting the field (10) into the dynamical equation for $w$ yields an infinite order equation for temporal evolution of the perturbation $\delta \tilde{w}$

$$
\begin{aligned}
\delta \dot{\tilde{w}}+ & \left\{\gamma+\frac{I_{0}}{6}\left[\bar{\kappa}+R\left(\kappa_{+}\left|e^{-i \sigma \Delta_{\perp}} e^{i \frac{3 \phi_{+}^{0}}{4} \delta \tilde{w}}\right|^{2}+\kappa_{-}\left|e^{-i \sigma \Delta_{\perp}} e^{-i \frac{3 \phi_{-}^{0}}{4} \delta \tilde{w}}\right|^{2}\right)\right]\right\} \tilde{w} \\
= & -\frac{I_{0} w_{h} R}{6}\left(\kappa_{+}\left|e^{-i \sigma \Delta_{\perp}} e^{i \frac{3 \phi_{+}^{0}}{4} \delta \tilde{w}}\right|^{2}+\kappa_{-}\left|e^{-i \sigma \Delta_{\perp}} e^{-i \frac{3 \phi_{-}^{0}}{4} \delta \tilde{w}}\right|^{2}-\bar{\kappa}\right) \\
& +\frac{\bar{\Delta} I_{0} u_{h} R}{3} \operatorname{Im}\left(e^{i \sigma \Delta_{\perp}} e^{-i \frac{3 \phi_{+}^{0}}{4} \delta \tilde{w}} e^{-i \sigma \Delta_{\perp}} e^{-i \frac{3 \phi_{-}^{0}}{4} \delta \tilde{w}}-1\right)-\frac{\bar{\Delta} I_{0} v_{h} R}{3} \operatorname{Re}\left(e^{i \sigma \Delta_{\perp}} e^{-i \frac{3 \phi_{+}^{0}}{4} \delta \tilde{w}} e^{-i \sigma \Delta_{\perp}} e^{-i \frac{3 \phi_{-}^{0}}{4} \delta \tilde{w}}-1\right) \\
& +\frac{I_{0} R}{9}\left(\frac{5}{2}-x_{h}\right)\left(\kappa_{+}\left|e^{-i \sigma \Delta_{\perp}} e^{i \frac{3 \phi_{+}^{0}}{4} \delta \tilde{w}}\right|^{2}-\kappa_{-}\left|e^{-i \sigma \Delta_{\perp}} e^{-i \frac{3 \phi_{-}^{0}}{4} \delta \tilde{w}}\right|^{2}-\Delta \kappa\right)
\end{aligned}
$$

where $\bar{\kappa}=\kappa_{+}+\kappa_{-}$and $\Delta \kappa=\kappa_{+}-\kappa_{-}$. From Eq. (12), we derive the dynamical equations for the perturbation amplitudes in the following way. First, we expand $e^{ \pm i \frac{3 \phi_{ \pm}^{0}}{4} \delta \tilde{w}}$, using (11), to second order in $\varepsilon$. After this, we evaluate $e^{-i \sigma \Delta_{\perp}} e^{ \pm i \frac{3 \phi_{ \pm}^{0}}{4} \delta \tilde{w}}$ and $e^{i \sigma \Delta_{\perp}} e^{ \pm i \frac{3 \phi_{ \pm}^{0}}{4} \delta \tilde{w}}$ by noting the propagation operator $e^{-i \sigma \Delta_{\perp}}$ is $e^{i \theta}$ in Fourier space. This means we can multiply the uniform $(q=0)$ terms by 1 , terms with wave vectors of length $q$ by $e^{i \theta}$, terms with wave vectors of length $\sqrt{3} q$ by $e^{3 i \theta}$, etc.; i.e., each term is an eigenfunction of the propagation operator. After this, the terms $\left|e^{-i \sigma \Delta_{\perp}} e^{ \pm i \frac{3 \phi_{ \pm}^{0}}{4}} \delta \tilde{w}\right|^{2}$ and $e^{i \sigma \Delta_{\perp}} e^{-i \frac{3 \phi_{+}^{0}}{4} \delta \tilde{w}} e^{-i \sigma \Delta_{\perp}} e^{-i \frac{3 \phi_{-}^{0}}{4} \delta \tilde{w}}$ are calculated, again by taking into account only expansion up to second order in $\varepsilon$. The calculation is simplified by our assumption that only terms resonant with $q_{c}$ are non-negligible, implicit in writing the ansatz (11) for $\delta \tilde{w}$. As we are here primarily interested in the existence of hexagonal solutions, we use the condition $\mathbf{q}_{1}+\mathbf{q}_{2}+\mathbf{q}_{3}=0$.

After equating the two sides of (12) and putting $\varepsilon=1$, we get the equations of the form

$$
\frac{d}{d t} A_{i}=\lambda A_{i}+\eta A_{j}^{*} A_{k}^{*}+\cdots
$$

for the amplitudes $A_{1}(t), A_{2}(t), A_{3}(t)$. Equations (13) describe mixing of modes on the same Talbot circle, to lowest orders in amplitude. They have the form of complex Ginzburg-Landau equations, common in many nonlinear systems $[37,48,53]$. The first term gives an exponential decrease or increase in amplitude of the transverse wave, with its vanishing determining the onset of instability. We regain here the threshold intensity of (8). The second (quadratic) term describes mixing of the three modes of a hexagon. It can easily be shown that positive (negative) hexagons are stable at threshold for $\eta>0(\eta<0)$, whereas stripes, squares, or rectangles are stable at threshold for $\eta=0[37,48]$. For the current purpose, it is sufficient to look for a single mode (i.e., stripes) as a representative for the inversion-symmetric, i.e., antiferromagnetic, state. In a more complete analysis, one could include an additional set of modes with wave vectors rotated by $90^{\circ}$ to include the square state, but this does not add any insight into the mechanism of the symmetry breaking from the antiferromagnetic to the ferrimagnetic states.
Upon inserting the critical diffractive phase shift of $\theta_{c}=\frac{\pi}{2}$, the coefficient $\lambda$ is given by relation (7) and $\eta$ is

$$
\begin{aligned}
\eta= & \left(\frac{3}{4}\right)^{2} \frac{R I_{t h} p}{2}\left\{\frac{8}{9}\left[\kappa_{+} \phi_{+}^{0}-\kappa_{-} \phi_{-}^{0}\right]\right. \\
& -\frac{w_{h}}{3}\left[\kappa_{+}\left(\phi_{+}^{0}\right)^{2}+\kappa_{-}\left(\phi_{-}^{0}\right)^{2}\right] \\
& +\frac{\bar{\Delta} u_{h}}{3}\left[\left(\phi_{+}^{0}\right)^{2}-\left(\phi_{-}^{0}\right)^{2}\right]+\frac{2 \bar{\Delta} v_{h}}{3} \phi_{+}^{0} \phi_{-}^{0} \\
& \left.+\frac{2}{9}\left[\frac{5}{2}-x_{h}\right]\left[\kappa_{+}\left(\phi_{+}^{0}\right)^{2}-\kappa_{-}\left(\phi_{-}^{0}\right)^{2}\right]\right\} .
\end{aligned}
$$

We now concentrate on the scan of threshold $\eta$ against $B_{z}$. In Fig. 10, we plot $\eta_{t h}$ against $B_{z}$ for two different $b_{0}$ values, corresponding to Strathclyde [Fig. 10(a)] and Nice [Fig. 10(b)] parameters. For $B_{z}=0$, we have $\eta_{t h}=0$, and the system is inversion symmetric, as also witnessed in the fact that $w_{h}^{t h}=0$ [see Fig. 9(d)]. For small $\left|B_{z}\right|$, there is a strong increase (decrease) in $\eta_{t h}$ for $B_{z}>0\left(B_{z}<0\right)$. This agrees with the results of both experiments and simulations for a spin-1 model. The behavior of $\eta_{t h}$ at small $\left|B_{z}\right|$ is determined by $w_{h}^{t h}$ and $v_{h}^{t h}$, giving a dispersive feature due to their coefficients in (14) being an even function in $B_{z}$ [and $w_{h}^{t h}, v_{h}^{t h}$ being odd in $B_{z}$; see Figs. 9(b) and 9(c)]. We note that simulations of the spin-1/2 model failed to produce hexagons at small $\left|B_{z}\right|$, indicating that the coupling to higher multipoles is indeed responsible for the symmetry breaking.
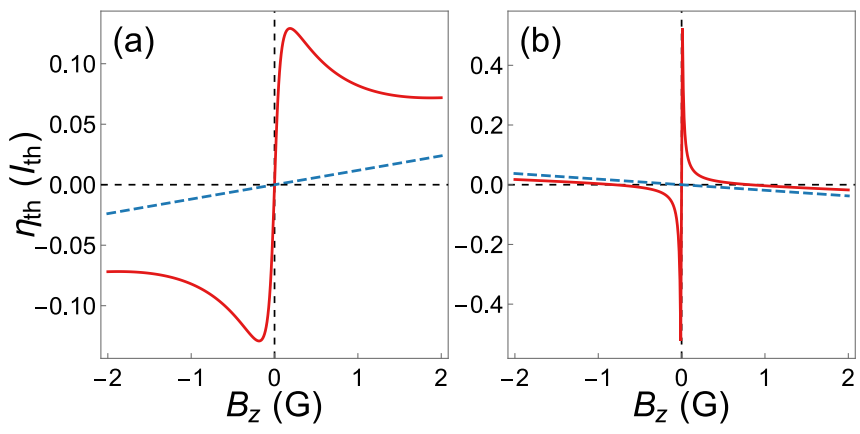

FIG. 10. Dependence of the threshold $\eta$ coefficient on $B_{z}$. (a) Parameters as in Fig. 8. (b) Parameters: $\Delta=-24 \Gamma_{2}, R=0.99, b_{0}=$ $110, \gamma=1 \times 10^{-4} \Gamma_{2}$. Red solid line: calculated from (14) for $p=1$. Blue dash-dotted line: calculated from the $w$-only model (see text). 
In zero $B$ field, the linearly polarized pump beam induces a $\Delta m=2$ coherence between the $m=1$ and $m=-1$ states via the $m^{\prime}=0$ excited state with the real part $u$ being pumped and the imaginary part $v$ being zero [see Figs. 9(a) and 9(b)]. The atomic coherence rotates in $B_{z}$ due to Larmor precession. This gives rise to the steady-state curves in Figs. 9(a) and 9(b) with an even shape for $u_{t h}$ and a dispersive (odd) shape for $v_{t h}$. This is the dominant origin of symmetry breaking. The variation of $w_{h}^{\text {th }}$ at small $\left|B_{z}\right|$ is due to coupling with $v_{h}^{\text {th }}$ [see Fig. 9(b)], given by the $-\frac{1}{6} P_{\Lambda+} \bar{\Delta} v$ term of the third equation of (1), whereas in a spin-1/2 model a symmetry breaking due to the incoherent Faraday effect is present (see dashed blue line) but much smaller than the effect due to the coupling to $v$. This coupling is a signature of coherent nonlinear Faraday rotation, and its physical origin will be explained at the end of this section.

At higher $\left|B_{z}\right|$, the magnitude of the $\eta$ coefficient starts to decrease, as both coherences $u, v$ are destroyed by the precession. For even higher fields, it starts to slowly increase again (for small $b_{0}$ ) or flips its sign (for large $b_{0}$ ). This qualitatively agrees with the experimental data, where a flipping of the direction of the hexagons was observed for large $b_{0}$. The sign reversal at high $b_{0}$ is present also in the spin-1/2 model. Its origin is in the competition of the three terms of the spin-1/2 model, where the last term [originating from the $\mathcal{D}(5 / 18-x / 9)$ term in the third Eq. of (1)] has a negative slope at large $B_{z}$ (and large negative $\bar{\Delta}$ ) and is responsible for the flipping. These slopes are determined by the incoherent linear and nonlinear Faraday effects, i.e., the variation of $\kappa_{ \pm}$and $\phi_{ \pm}^{0}$ with $B_{z}$. The physical interpretation of the said competition is still under investigation.

We note that in previous experiments in spin-1/2 sodium vapors a change of inversion symmetry was not observed with varying a longitudinal magnetic field alone [50,52,54], in line with the theoretical treatment given here, as the incoherent Faraday effect is very small for the pressure broadened transition under study in Refs. [50,52,54]. A symmetry-breaking transition was only obtained in a transverse magnetic field $[50,54]$ or by perturbing the input polarization to be elliptical $[29,30]$. In the former case, the interplay of dark state pumping of $w_{h}$ and spin flips in the transverse field is influenced by not only the Zeeman shifts but also the light shift changing the ground-state degeneracy, and the effect was accompanied by a large asymmetry in the absorption of the two circular light components. Such large absorption asymmetry was not observed for our system parameters at small $B_{z}$, either in experiments or simulations. In the case of elliptical input polarization, the symmetry of optical pumping is obviously broken. We expect a similar effect in the cold-atom system but did not investigate it further as the analogy between magnetic ordering via light-induced interactions and in condensed matter systems, respectively, simple models for magnetism, is better worked out by changing the magnetic field than the input polarization.

\section{Coupling of magnetic multipoles}

We now explain the physical mechanisms for breaking of inversion symmetry and increase of threshold at small $\left|B_{z}\right|$ in the simplified model used throughout this section. It is well known that quantum interference effects can influence the steady state of a laser-driven system in a $\Lambda$ (-like) configuration, depending on the phase of the ground-state coherence density matrix element [55]. It is thus natural to expect that the change of the values of $u_{h}$ and $v_{h}$ should influence our results at small $\left|B_{z}\right|$.

The change of symmetry at small $\left|B_{z}\right|$ was already seen to occur due to coupling of $w_{h}$ and $v_{h}$. To see the origin of this coupling, we will switch to the usual representation of density matrix elements in the Zeeman sublevel basis. For simplicity, we put $\Omega_{+}=\Omega_{-}=\Omega_{0}, \Omega_{0} \in \mathbb{R}$ (i.e., beam is linearly polarized along $y^{\prime}$ ). We are interested in coupling of the optical coherence $\rho_{10}$ (where the apostrophe denotes a sublevel of the excited state) to the population $\rho_{11}$ and atomic coherence $\rho_{1-1}$. After adiabatic elimination, the optical coherence is given by

$$
\rho_{10^{\prime}}=\frac{\Omega_{0}}{6} \frac{\rho_{11}+\rho_{1-1}}{i \Gamma_{2}-\Delta},
$$

where we have here neglected $\Omega_{z}$ with respect to $\Delta$ in the denominator (as for small $B_{z},|\Delta| \gg\left|\Omega_{z}\right|$ ) and we note that the same expression appears in a $\Lambda$ system (as given, e.g., in [56], apart from the sign convention and with excited-state population here being neglected). We also note that for putting $\Omega_{+}=0$ and keeping $\Omega_{-}=\Omega_{0}$, the $\rho_{1-1}$ term in (15) vanishes. The relevant term in the dynamical equation for $\rho_{11}$ is

$$
\dot{\rho}_{11} \propto 2 \Omega_{0} \operatorname{Im}\left(\rho_{10^{\prime}}\right) .
$$

The $\rho_{11}$ term in (15) is due to population leaving the state with $m=1$ and is contained in the decay rate $\Gamma_{w}$ of (1). Keeping only the $\rho_{1-1}$ term, (16) is now

$$
\dot{\rho}_{11} \propto-\frac{I_{0}}{3} \bar{\Delta} \operatorname{Im}\left(\rho_{1-1}\right),
$$

where we have neglected the $\Gamma_{2}$ term with respect to the $\Delta$ term as it cancels out in the $w$ equation (but not for $x$; see below). The dynamical equation for $\rho_{-1-1}$ has the same dependence on $\operatorname{Im}\left(\rho_{1-1}\right)$ but with a positive sign, which gives the $-\frac{1}{6} \bar{\Delta} P_{\Lambda+} v$ term in the $w$ equation of (1). For a finite $v$, an optical coherence in the $\Lambda$ configuration can thus give rise to optical pumping of a stretched state with $m= \pm 1$, depending on the sign of $v$. We interpret this process as coherent two-photon Raman pumping.

The importance of light-induced $|\Delta m|=2$ Zeeman coherences for nonlinear Faraday rotation in an $F \rightarrow F+1$ (with $F \geqslant 1$ ) transition was noted in Refs. [36,44], where amplitude modulation of light [57] was used to detect narrow resonances in the demodulated in-phase rotation signals. Resonances at twice the Larmor frequencies equal to intensity modulation frequencies were interpreted to arise due to beating of the oscillating light-induced Zeeman coherences and Larmor precession caused by a longitudinal magnetic field. By writing and solving Eqs. (1), the experimental results of these papers are corroborated and their theoretical analysis made more concrete, albeit in a simpler level structure, expected to exhibit equivalent behavior.

Increase of threshold intensity with increasing $\left|B_{z}\right|$ at small $\left|B_{z}\right|$ is a consequence of the reduction of population in the $m=$ 0 state, in (1) caused by the coupling of $x_{h}$ and $u_{h}$. The origin 
of this coupling is the excited-state population $\rho_{0^{\prime} 0^{\prime}}$, which is in our derivation of (1) adiabatically eliminated but still "feeds" the ground-state populations. Using the assumptions made above, one gets for the coupling of the excited-state population in $m^{\prime}=0$ and the ground state $|\Delta m|=2$ coherence

$$
\rho_{0^{\prime} 0^{\prime}} \propto \frac{I_{0}}{3 \Gamma_{1}} \operatorname{Re}\left(\rho_{1-1}\right),
$$

as is apparent also from the equations of Ref. [56] for a $\Lambda$ system. The above expression indicates that a large (small) value of $u$ leads to large (small) population of the excited state with $m^{\prime}=0$. The optical coherences $\rho_{10^{\prime}}$ and $\rho_{-10^{\prime}}$ are also affected by $\operatorname{Re}\left(\rho_{1-1}\right)$, which leads to the following terms in the dynamical equations for $\rho_{11}$ and $\rho_{-1-1}$,

$$
\dot{\rho}_{11}, \dot{\rho}_{-1-1} \propto-\frac{I_{0}}{3} \operatorname{Re}\left(\rho_{1-1}\right),
$$

which together with (18) leads to the $-\frac{1}{3} P_{\Lambda+} u$ term in the fourth equation of (1). Since $u$ decreases with increasing $\left|B_{z}\right|$ at small $\left|B_{z}\right|$, the $\rho_{0^{\prime} 0^{\prime}}$ will also decrease. The $m=0$ population relative to the total stretched state populations will likewise decrease, since the probability of decay of $\rho_{0^{\prime} 0^{\prime}}$ into $m=0$ is two times greater than for decay into $m=1$ and $m=-1$ together [see Fig. 5(a)]. This decrease of relative population in $m=0$ as $\left|B_{z}\right|$ grows from zero to a small value leads hence to an increase in $x$. This then leads to an increase in the threshold intensity, as explained in Sec. VIA [see Eq. (9)].

\section{CONCLUSION}

We have studied the properties of transverse spin patterns in a cold atomic cloud of ${ }^{87} \mathrm{Rb}$ subject to laser driving with mirror feedback. Experimental scans of pattern properties against longitudinal magnetic field were compared to the mostly analytical results of our spin-1 theoretical model. Inversion-symmetric antiferromagnetic spin patterns give way to ferrimagnetic patterns in a weak longitudinal magnetic field. It was worked out that the inversion symmetry of the system, governing the pattern symmetries, is broken for small magnetic fields by coupling of the dipole magnetic polarization of the atoms to the $|\Delta m|=2$ ground state coherence precessing in the $B_{z}$ field. This is consistent with the conclusion of Faraday rotation studies performed in a cold atomic cloud with similar level structure [36]. The increase of threshold intensity for pattern formation with small magnetic field is in our model a result of reduced pump rates into the stretched states caused by the coupling of the quadrupolar polarization components, whereas perturbations in the higher order magnetic moments are responsible for threshold reduction at larger magnetic fields. The spin- 1 model thus accounts for the experimental dependence of pattern properties on the longitudinal magnetic field, exhibiting dependence on both dipole and quadrupole magnetic components of the density matrix expansion. This constitutes a step beyond previous work on spin-1/2 models [8,27,29,30,50,54]. Optical interference in $\mathrm{Rb}$ vapors with multilevel structure has recently been employed to observe interesting linear [58] and nonlinear [59] optical effects.
Our cold-atom setup has some analogy with the Ising model, where interactions are light mediated over a range determined by diffractive dephasing, and the lattice emerges spontaneously as opposed to it being set externally [25]. The work is part of a relatively recent research effort of using laser light as an atomic interaction vector, due to its easy controllability and extremely weak decoherence of its states during propagation. In recent years various setups, from cavities to nanoscopic solid state devices, have been employed to engineer photon-mediated interactions between atoms for a wide range of quantum technological purposes [60-62]. Self-organization phenomena in driven systems have for a long time played an important technological role, from the invention of the laser [63] to recent promising applications in frequency combs [64] and chemical engineering [65]. It is thus interesting to ask whether and how self-organization will find its application in next-generation quantum technologies. The SFM setup may offer some advantages in this respect, and we will continue to explore its quantum technological potential with both thermal and degenerate cold atoms. For example, Ref. [25] reports indication of a hysteretic firstorder phase transition between the unstructured and the ferrimagnetic state, opening the exciting possibility to study nucleation phenomena and localized magnetic states. Also, by incorporating different geometries in the feedback part of the setup, e.g., by using a spatial light modulator, we expect to be able to engineer different forms of light-mediated interactions, which is a very attractive feature for quantum simulation.

Although the pattern length scale is here set by the mirror distance and the allowed symmetry at threshold is set by $B_{z}$, the transverse pattern spin modes are degenerate in the sense that a pattern realization with any orientation and center position is equally probable. This multimode situation is inherent in the SFM setup and arises due to transverse rotational and translational symmetries of the initial system. Light-mediated self-organization of atomic degrees of freedom in multimode configurations is currently generating some interest, with possible broader implications for the field of condensed-matter physics [4-13]. In addition to the optomechanical effects, spinor effects have sparked interest in this community as well $[15,18]$.

\section{ACKNOWLEDGMENTS}

The Strathclyde group is grateful for support from the Leverhulme Trust. I.K. gratefully acknowledges a University Studentship from the University of Strathclyde and Postdoctoral Fellowships from the Croatian Science Foundation (Project Frequency-Comb-Induced Optomechanics No. IP2014-09-7342) and the Scholarship Foundation of the Republic of Austria. The Nice group acknowledges support from CNRS, UNS, and Région PACA. The collaboration between the Strathclyde and Nice groups was supported by the Royal Society (London), CNRS and in particular the Laboratoire International Associé "Solace," and the Global Engagement Fund of the University of Strathclyde. The final stage of this work was performed in the framework of the European Training network ColOpt, which is funded by the European Union's Horizon 2020 research and innovation programme under the Marie Sklodowska-Curie action, Grant Agreement No. 721465 . 
[1] I. D. Leroux, M. H. Schleier-Smith, and V. Vuletic, Phys. Rev. Lett. 104, 073602 (2010).

[2] M. H. Schleier-Smith, I. D. Leroux, and V. Vuletić, Phys. Rev. Lett. 104, 073604 (2010).

[3] M. A. Norcia, R. J. Lewis-Swan, J. R. Cline, B. Zhu, A. M. Rey, and J. K. Thompson, Science 361, 259 (2018).

[4] S. Gopalakrishnan, B. L. Lev, and P. M. Goldbart, Phys. Rev. A 82, 043612 (2010).

[5] G. Labeyrie, E. Tesio, P. M. Gomes, G.-L. Oppo, W. Firth, G. Robb, A. Arnold, R. Kaiser, and T. Ackemann, Nat. Photon. 8, 321 (2014).

[6] E. Tesio, G. R. M. Robb, T. Ackemann, W. J. Firth, and G.-L. Oppo, Phys. Rev. Lett. 112, 043901 (2014).

[7] Y.-C. Zhang, V. Walther, and T. Pohl, Phys. Rev. Lett. 121, 073604 (2018).

[8] B. L. Schmittberger and D. J. Gauthier, New J. Phys. 18, 103021 (2016).

[9] S. Ostermann, F. Piazza, and H. Ritsch, Phys. Rev. X 6, 021026 (2016).

[10] I. Dimitrova, W. Lunden, J. Amato-Grill, N. Jepsen, Y. Yu, M. Messer, T. Rigaldo, G. Puentes, D. Weld, and W. Ketterle, Phys. Rev. A 96, 051603(R) (2017).

[11] G. R. M. Robb, E. Tesio, G.-L. Oppo, W. J. Firth, T. Ackemann, and R. Bonifacio, Phys. Rev. Lett. 114, 173903 (2015).

[12] J. Léonard, A. Morales, P. Zupancic, T. Esslinger, and T. Donner, Nature (London) 543, 87 (2017).

[13] F. Mivehvar, S. Ostermann, F. Piazza, and H. Ritsch, Phys. Rev. Lett. 120, 123601 (2018).

[14] J. Léonard, A. Morales, P. Zupancic, T. Donner, and T. Esslinger, Science 358, 1415 (2017).

[15] M. Landini, N. Dogra, K. Kroeger, L. Hruby, T. Donner, and T. Esslinger, Phys. Rev. Lett. 120, 223602 (2018).

[16] A. J. Kollár, A. T. Papageorge, V. D. Vaidya, Y. Guo, J. Keeling, and B. L. Lev, Nat. Commun. 8, 14386 (2017).

[17] S. C. Schuster, P. Wolf, D. Schmidt, S. Slama, and C. Zimmermann, Phys. Rev. Lett. 121, 223601 (2018).

[18] R. M. Kroeze, Y. Guo, V. D. Vaidya, J. Keeling, and B. L. Lev, Phys. Rev. Lett. 121, 163601 (2018).

[19] T. Keller, V. Torggler, S. B. Jäger, S. Schütz, H. Ritsch, and G. Morigi, New J. Phys. 20, 025004 (2018).

[20] W. J. Firth, J. Mod. Opt. 37, 151 (1990).

[21] L. Lugiato, F. Prati, and M. Brambilla, Nonlinear Optical Systems (Cambridge University Press, Cambridge, UK, 2015).

[22] M. Tamburrini, M. Bonavita, S. Wabnitz, and E. Santamato, Opt. Lett. 18, 855 (1993).

[23] T. Ackemann and W. Lange, Phys. Rev. A 50, R4468 (1994).

[24] A. Camara, R. Kaiser, G. Labeyrie, W. J. Firth, G.-L. Oppo, G. R. M. Robb, A. S. Arnold, and T. Ackemann, Phys. Rev. A 92, 013820 (2015).

[25] I. Krešić, G. Labeyrie, G. R. M. Robb, G.-L. Oppo, P. M. Gomes, P. Griffin, R. Kaiser, and T. Ackemann, Commun. Phys. 1, 33 (2018).

[26] G. Labeyrie, I. Krešić, G. R. M. Robb, G.-L. Oppo, R. Kaiser, and T. Ackemann, Optica 5, 1322 (2018).

[27] F. Mitschke, R. Deserno, W. Lange, and J. Mlynek, Phys. Rev. A 33, 3219 (1986).

[28] G. Grynberg, Opt. Commun. 109, 483 (1994).
[29] A. J. Scroggie and W. J. Firth, Phys. Rev. A 53, 2752 (1996).

[30] A. Aumann, E. Büthe, Y. A. Logvin, T. Ackemann, and W. Lange, Phys. Rev. A 56, R1709 (1997).

[31] B. L. Schmittberger and D. J. Gauthier, JOSA B 33, 1543 (2016).

[32] G. A. Muradyan, Y. Wang, W. Williams, and M. Saffman, in Nonlinear Guided Waves and Their Applications (Optical Society of America, 2005), p. ThB29.

[33] J. A. Greenberg, B. L. Schmittberger, and D. J. Gauthier, Opt. Express 19, 22535 (2011).

[34] B. L. Schmittberger and D. J. Gauthier, Phys. Rev. A 90, 013813 (2014).

[35] J. Dalibard and C. Cohen-Tannoudji, JOSA B 6, 2023 (1989).

[36] A. Wojciechowski, E. Corsini, J. Zachorowski, and W. Gawlik, Phys. Rev. A 81, 053420 (2010).

[37] M. C. Cross and P. C. Hohenberg, Rev. Mod. Phys. 65, 851 (1993).

[38] E. Ciaramella, M. Tamburrini, and E. Santamato, Appl. Phys. Lett. 63, 1604 (1993).

[39] M. A. Neto and J. R. de Sousa, Phys. Lett. A 330, 322 (2004).

[40] T. Kaneyoshi, J. Magn. Magn. Mat. 406, 83 (2016).

[41] P. Kushwaha, R. Rawat, and P. Chaddah, J. Phys.: Condens. Matter 20, 022204 (2008).

[42] S. M. Yusuf, N. Thakur, M. Medarde, and L. Keller, J. Appl. Phys. 112, 093903 (2012).

[43] W. J. Firth, I. Krešić, G. Labeyrie, A. Camara, and T. Ackemann, Phys. Rev. A 96, 053806 (2017).

[44] W. Gawlik and A. Wojciechowski, A. Opt. Spectrosc. 111, 626 (2011)

[45] I. Krešić, Ph.D. thesis, University of Strathclyde, Glasgow, United Kingdom, 2016.

[46] M. Auzinsh, D. Budker, and S. Rochester, Optically Polarized Atoms: Understanding Light-Atom Interactions (Oxford University Press, Oxford, UK, 2010).

[47] M. Fleischhauer, A. Imamoglu, and J. P. Marangos, Rev. Mod. Phys. 77, 633 (2005).

[48] G. D'Alessandro and W. J. Firth, Phys. Rev. A 46, 537 (1992).

[49] R. Neubecker, G.-L. Oppo, B. Thuering, and T. Tschudi, Phys. Rev. A 52, 791 (1995).

[50] Y. A. Logvin, A. Aumann, M. Tegeler, T. Ackemann, and W. Lange, J. Opt. B: Quantum Semiclassical Opt. 2, 426 (2000).

[51] H. F. Talbot, London Edinburgh Phil. Mag. J. Sci. 9, 401 (1836).

[52] A. Aumann, Ph.D. thesis, Westfalische Wilhelms-Universität, Muenster, Germany, 1999.

[53] I. S. Aranson and L. Kramer, Rev. Mod. Phys. 74, 99 (2002).

[54] A. Aumann, E. G. Westhoff, T. Ackemann, and W. Lange, J. Opt. B: Quantum Semiclassical Opt. 2, 421 (2000).

[55] J. P. Marangos, J. Mod. Opt. 45, 471 (1998).

[56] R. G. Brewer and E. L. Hahn, Phys. Rev. A 11, 1641 (1975).

[57] W. Gawlik, L. Krzemień, S. Pustelny, D. Sangla, J. Zachorowski, M. Graf, A. Sushkov, and D. Budker, Appl. Phys. Lett. 88, 131108 (2006).

[58] Z. Zhang, Y. Zhang, J. Sheng, L. Yang, M.-A. Miri, D. N. Christodoulides, B. He, Y. Zhang, and M. Xiao, Phys. Rev. Lett. 117, 123601 (2016).

[59] Z. Zhang, F. Wen, J. Che, D. Zhang, C. Li, Y. Zhang, and M. Xiao, Sci. Rep. 5, 15058 (2015). 
[60] H. Ritsch, P. Domokos, F. Brennecke, and T. Esslinger, Rev. Mod. Phys. 85, 553 (2013).

[61] G. Kurizki, P. Bertet, Y. Kubo, K. Mølmer, D. Petrosyan, P. Rabl, and J. Schmiedmayer, Proc. Natl. Acad. Sci. USA 112, 3866 (2015).

[62] D. E. Chang, J. S. Douglas, A. González-Tudela, C.-L. Hung, and H. J. Kimble, Rev. Mod. Phys. 90, 031002 (2018).
[63] A. L. Schawlow and C. H. Townes, Phys. Rev. 112, 1940 (1958).

[64] T. J. Kippenberg, A. L. Gaeta, M. Lipson, and M. L. Gorodetsky, Science 361, eaan8083 (2018).

[65] Z. Tan, S. Chen, X. Peng, L. Zhang, and C. Gao, Science 360, 518 (2018). 